\title{
Services and a Distributed Ecosystem for Supporting Mobile Medical Application Development
}

\section{Petrenko Al*}

National Technical University, Kyiv Sikorsky Polytechnic Institute, Ukraine

\begin{abstract}
The main objective of this paper is to provide novel methods facilitating an efficient access and reuse of healthcarerelated data sources within a large scalable and reliable infrastructure, so called ecosystem of ontology-based services which reflect big data and specific patient care. This task focuses on developing ontology-based services for gathering outputs from the various data sources (portable wearable devices, the EHR data, clinical notes, claims, medical imaging data, etc.), that are used in various applied fields of mHealth, and on creating management technology for provision of interconnected healthcare-related data sources and processing procedures. The developed services are the main 'building blocks' for more complex applications that use some medical data processing scenarios. To hide the low-level details of the service orchestration mechanism, an automated ontology-based workflow management engine is introduced, that will allow expressing various data processing workflows in the abstract terms of the 'glue' high-level ontology rather than in low-level details of interaction different services.
\end{abstract}

Keywords: mHealth; self-care; Personal Health Systems; mobile phone; SOC; EDA; Cloud services; decision support systems; Web-services

\section{Introduction}

E-Health promotion is a priority of the European Commission. Many specialists in the world believe that patient empowering can transform medical care. Wireless Internet connectivity, cloud computing, mobile devices (smartphones and tablets), mobile applications and sensors modernized clinical trials, internet connectivity, advanced diagnostics, targeted therapies, and other science enable the individualization of medicine and force overdue radical change in how medicine is delivered, as well as regulated and reimbursed. Mobile devices are being used to capture data at the point of care and to keep the lines of communication open no matter where the doctor is, and they're being used at home to record and send vital health data back to the health professional and, in turn, to send important healthcare management information back to the patient. The personal data is used to track the ups and downs of patient's conditions as they go about their lives.

Healthcare was once about trying to treat a sick patient. Healthcare organizations around the world now have an opportunity to shift this focus to one of keeping the public healthy and anticipating health issues before they become a problem. Physicians and other health professionals seek to monitor patients remotely through new technologies, aiming to identify problems early and cut costs and inefficiencies in the healthcare system. Identifying people at risk of becoming ill or developing a serious condition and providing the foresight to prescribe preventive measures is a very real possibility.

Improved qualities of care, better clinical outcomes are the observable benefits of this future medical care revolution as it was declared in the GREEN PAPER on mobile Health [1], the Action Plan 2012-2020 [2], and recommendations of the eHealth Stakeholder Group [3], the EU eHealth Governance Initiative [4], and the eHealth Task Force [5].

One-stop shop for health information can be created by building a smart healthcare ecosystem where patients' health parameters can be permanently monitored by the networked medical heterogeneous devices and solutions, and then used for decision making together with the structured EHR data, unstructured clinical notes, medical imaging data, etc. Effectively integrating and efficiently analysing various forms of healthcare data over a period of time can answer many of the impending healthcare problems.
Let's imagine that every medical sensor (or another data resource) of that ecosystem has its own URI allowing doctors and patients interact with it via the web browser, and at the same time every sensor can have the software interface - a set of web services allowing intelligent software agents to interact with it (analyse the data etc.) on behalf of doctors and patients. Certainly the integration of that with the classical medical record is vital.

Current health data is diverse, comprising structured and unstructured information in a range of formats. It is vitally important for health care organizations to get the tools, infrastructure, and techniques in place now to deal with big data in effective way. By the diversity of the participating devices we should provide the integration framework for Patient Monitoring (both healthy and sick patients) and personalizing healthcare for every patient.

EHRs and automation tools already exist to identify and stratify individual patients who need special attention or care. But there is a lack of semantic interoperability, no standards exist on interoperability of health care data, there are only syntactic standards (e.g. HL7 from the U.S.). Although there are ongoing efforts to develop OWLDL ontology to solve HL7 v.2 and v.3 interoperability problem, still there are no adopted solutions. A draft standard, FHIR (Fast Health Interoperability Resources), the younger sibling of interoperability standard HL7, is already being used by developers, but the development of ontology for FHIR is still urgent [6].

We propose to use semantically-enhanced event-driven serviceoriented architectural model (SEMSOA) to develop an open ecosystem (based on the Open Science Commons) for providing wider interoperability of healthcare services for patients' groups with different illnesses and for supporting a policy of standardization of

*Corresponding author: Petrenko Al, National Technical University, Kyiv Sikorsky Polytechnic Institute, Ukraine, Tel: 38044 2367989; E-mail: petrenko@cad.kiev.ua

Received September 14, 2016; Accepted September 19, 2016; Published September 30, 2016

Citation: Petrenko Al (2016) Services and a Distributed Ecosystem for Supporting Mobile Medical Application Development. J Telecommun Syst Manage 5: 140. doi: 10.4172/2167-0919.1000140

Copyright: () 2016 Petrenko Al. This is an open-access article distributed under the terms of the Creative Commons Attribution License, which permits unrestricted use, distribution, and reproduction in any medium, provided the original author and source are credited. 
these healthcare services. Events in this ecosystem can be initiated by patients, doctors, and devices outcomes. Due to such ecosystem, healthcare is migrating from episodic and fragmented illness response to a patient-centric model of care delivery.

We are going to develop the repository of services related to processes of data collection and storing from all data sources (and developing the ontology of these services). This repository will include:

- Ontology-based services for gathering the various portable wearable devices output data.

- Ontology-based services for managing the EHR data, clinical notes, claims, medical imaging data, etc.

- Ontology-based services for capturing the information of patients' behaviour.

- Services for identifying patients at risk of becoming ill or developing a serious condition by constant patients' monitoring and personalizing healthcare.

- Services for insights synthesis and information of patients, health professionals, health payers and insurers, and life science companies actions.

- Services for aggregating individual patients' data across a community into a broader, meaningful view of health and healthcare in a particular region to support healthcare migrating from episodic and fragmented illness response to a patient-centric model of public healthcare.

The mechanism of customer-driven medical applied software development will be provided by compositing and orchestrating dynamically discovered services from developed repository to form the individual patient pathway (patient-specific workflows) of monitoring and treatment, taking into account different existing rules, regulations and standards.

We propose also to establish connection between data sources, patients, doctors and healthcare organizations by developing a healthcare ecosystem in the Cloud for data sharing across the entire ecosystem, using SaaS and IaaS technologies to host eHealth community-support services, services for analytic processing of data across the healthcare ecosystem. The solutions will be offered to large and medium size healthcare organizations, small research communities, and the long-tail of science, education, industry and SMEs. Consequently, these customers will be able to manage patient populations to improve health, improve outcomes and reduce costs by identification of patients with the same patient's characteristics.

Expanding solutions across the health continuum, from healthy living, prevention, diagnosis, treatment, recovery and home care, to truly impact patients' health at the individual and population levels and to the most clinically and cost-effective treatments to apply. Even more, by analysing doctors' prescriptions and comparing them against medical guidelines it is possible to decrease treatment error rates and liability claims, especially those arising from clinical mistakes, and, perhaps, to find more effective approaches to the treatment of different conditions.

We are going to apply secure cloud computing technologies such as virtual desktop infrastructure which allows practitioners easy access to patient data without ever storing protected health information on mobile or remote devices such as smartphones, tablets and laptops, besides making broader using of encryption, bolstering access control, authentication and access tracking.
The proposed healthcare ecosystem may be used not only for expert functions but also for modelling of developing health systems in different countries. As result some dynamic innovative healthcare model can be established, which will take into account both the current technical capabilities, and emerging technologies. This will allow quick response to the market dynamics and offer the developers a system of innovation implementation, to provide consulting services for the implementation of new healthcare systems in various markets, hold the bulk of the information in this area. Having the centralized repository of services and data, it's possible to develop a universal adapting software product to embed software solutions from third parties that is compatible with a variety of sensors and to provide the ability to get medical help in any place of Europe.

\section{Related Work}

Healthcare has slowly been progressing through three stages of data management: data collection, data sharing, and data analytics. In healthcare, the first phase is characterized by widespread Electronic Health Record (EHR) adoption. In the second stage, the need for sharing data among members of the workflow team becomes apparent. In the case of a healthcare, this phase is characterized by health information exchange. Healthcare is now entering the third stage, the data analysis phase, which shall be characterized by the adoption of Enterprise Data Warehouses (EDW), when the data collected and shared, can be used to analyze aspects of the workflow that are reflected in the patterns of the aggregated data.

The drive now is to understand about a patient as much as possible and at their earliest life stages possible - hopefully picking up the warning signs of a serious illness at an early stage so the that treatment is far more simple (and less expensive) than when it is spotted at a later stage. The aim is to collect data from various sources (such as medical and insurance records, wearable sensors, medical and healthcare mobile applications, genetic data and even social media use) to draw a comprehensive picture of the patient as of an individual, in order to offer a tailored healthcare package. This requires the capability to link data and extract potentially valuable information from unstructured data in an automated cost-effective way by applying mobile applications, disease management applications, social integration platforms, broad band communication, Internet of things, cloud computing, coaching programs.

Although healthcare systems' organization is a national or regional competence, it focuses on cross-border European-wide issues and on possible coordinated actions at EU level.

To reach this the special study SMART 2007/0059 was launched by European Commission in 2008-2009 years for creating the legal framework for interoperable electronic health services in Europe. The study also provided the analysis of each member healthcare state [7] It facilitated comparison and potential bringing together of all the national legislations in the EU Member States and provided details of 10 good practice examples (say, the Barcelona Telemedicine Clinic provides services to Swedish and Danish hospitals).

The Project SMART 2007/0059 has shown that level of ICTmaturity of the healthcare sector differs considerably from one Member State to another. The conclusion was that the health sector in Europe necessarily needs to be seen as a very fragmented landscape and that this fragmentation will not be eliminated in the near future. A European regulatory framework for eHealth should therefore not only take this diversity into account but it should fundamentally be based on it. 
A further obstacle for the delivery of cross-border eHealth services is the divergence in the liability regimes. If a patient wishes to make use of cross-border eHealth services delivered by a provider established in another Member State, she/he will immediately be confronted with uncertainty about the applicable rights as a patient. Cross-border delivery of eHealth services can be facilitated if providers don't necessarily have to register or apply for a license in every Member State separately.

Another project $e$-SENS (Electronic Simple European Networked Services) was launched by the European Commission in April 2013 with 36 months duration. By developing technical components for seamless electronic communication, this project facilitates the provision of cross-border digital services in a number of policy domains: e-Justice, business start-up, e-ID, e-Procurement, and e-Health [8]. It is based on the EC study SMART 2011/0074 on the Analysis of the Needs for Cross-Border Services and Assessment of the Organizational, Legal, Technical and Semantic Barriers, which validates the key cross-border public services with the highest impact that should be deployed; identifies the barriers that need to be overcome; points to the potential benefits of the cross-border public services implementation; and provides scenarios for a discussion on if and how these services could have been implemented by 2015 [9].

The project e-SENS will develop the digital infrastructure for improving the quality of public services in EU. It will support the implementation of European policies, in particular the Digital Agenda for Europe in accordance with the Action 91 of the Digital Agenda: "Member States to agree a common list of key cross-border public services" which will include also the identification of desirable crossborder services for eHealth applications, particularly, in the areas of electronic health records, telemedicine and e-prescription [10]. In 2013 the European Commission also initiated the development of a common eHealth EU Interoperability Framework (EIF), which can help expand a digital single market for healthcare, opening up a competition and reducing costs for future reinvestments to update healthcare systems [11]. The study defines a vision of the EU eHealth Interoperability Framework at four levels: technical, semantic, organizational and legal. It also assesses some technical specifications from two development organizations Integrating the Healthcare Enterprise (IHE) [12] and Continua Health Alliance [13], and epSOS project (European Patients Smart open Services) against the identification criteria of the annex II of the Regulation on European Standardization [14]. In particular, epSOS project (2012-2014) was aimed to design, build and evaluate a service infrastructure that demonstrates cross-border interoperability between electronic health record systems in Europe. The epSOS was focused on participating partner countries exchange of patient prescription and electronic health record summaries.

SUSTAINS (Support Users To Access Information and Services) is a three-year project aimed at developing and deploying a basket of services in 11 European regions providing patients' access to Electronic Health Records (EHR) [15]. Although each of these services has a specific objective, all the services contribute to the achievement of a new paradigm in healthcare in which the citizen/patient is no longer a passive subject, but an active player in the management of his/her own health. Although each of these services has a specific objective, all the services contribute to the achievement of a new paradigm in healthcare in which the citizen/patient is no longer a passive subject, but an active player in the management of his/her own health.

The United4Health (Universal solutions in Telemedicine Deployment for European HEALTH care) aims to exploit and roll- out innovative telemedicine solutions previously validated as part of the European Renewing Health project in the treatment of patient with diabetes, chronic obstructive pulmonary or cardiovascular diseases [16,17]. These solutions will allow patients to manage their disease to adjust the choice and dose of medicines, promote treatment compliance, help professionals to detect early signs of deterioration and thus contribute to the sustainability of the health system.

The implemented United4Health was successfully deployed within the secured Norwegian Health Network [16]. United4Health is partially funded under the ICT Policy Support Programme (ICT PSP) as part of the Competitiveness and Innovation Framework Programme by the European Community. Finally, SemanticHealthNet (SHN) Project (2012-2015) is worth to be referred as it has improved semantic interoperability of the content of electronic health records (EHRs) by providing an integrative semantic abstraction on top of them [18]. This approach has been demonstrated on chronic heart failure and cardiovascular prevention but it targets the whole range of healthrelated information about all medical domains. Results of e-SENS, EIF, epSOS and SHN will be used to specify the deployment of cross-border eHealth services in the frame of the Connection Europe Facility (CEF) [19], but they will also potentially be used for national, regional or project based deployments.

It is worth to mention that Europe does not yet reap the maximum benefit from Big Data technologies and eHealth interoperability. Weaknesses in standard-setting, public procurement and coordination between public authorities prevent digital services and devices used by Europeans from working together as well as they should. Projects are needed to support upper levels of health analytic technology which include: Personalized Medicine and Prescriptive Analytics (Level 8), Clinical Risk Intervention and Predictive Analytics (Level 7), and Population Health Management Suggestive Analytics (Level 6), preceded levels of Waste and Care Variability Reduction, External Reporting, Automated Internal Reporting, Standardized Vocabulary Patient, and Enterprise Data Warehouse [20].

There were some attempts to create a one-stop shop for health information. Such task is very difficult and even Google Health was an early attempt at integration that failed because uploading the data was a hassle [21]. Microsoft was more successful with its HealthVault (the web-based electronic health records platform), which is a trusted place for people to gather, store, use and share online health information that patients have uploaded themselves [22]. IBM recently announced that its Watson system was going to focus on the healthcare field (i.e., supporting diagnosis and treatment) $[23,24]$. IBM has estimated that almost 5 million people around the world will be connected to their healthcare providers.

\section{Activity Overview}

\section{Concept}

Our proposal is the semantically-enhanced event-driven serviceoriented architectural model (SEMSOA) for the Cloud eHealth ecosystem, which can facilitate the development of the intelligent software agents discovering and interacting with heterogeneous devices and data (Figure 1). In other words, the framework will hide low-level details of interaction with specific web services representing concrete devices and their data the means of semantic middle layer. SEMSOA-powered platform should help to minimize the efforts in the development of the new medical software capable to inter-operate with the joined cloud of networked devices. 


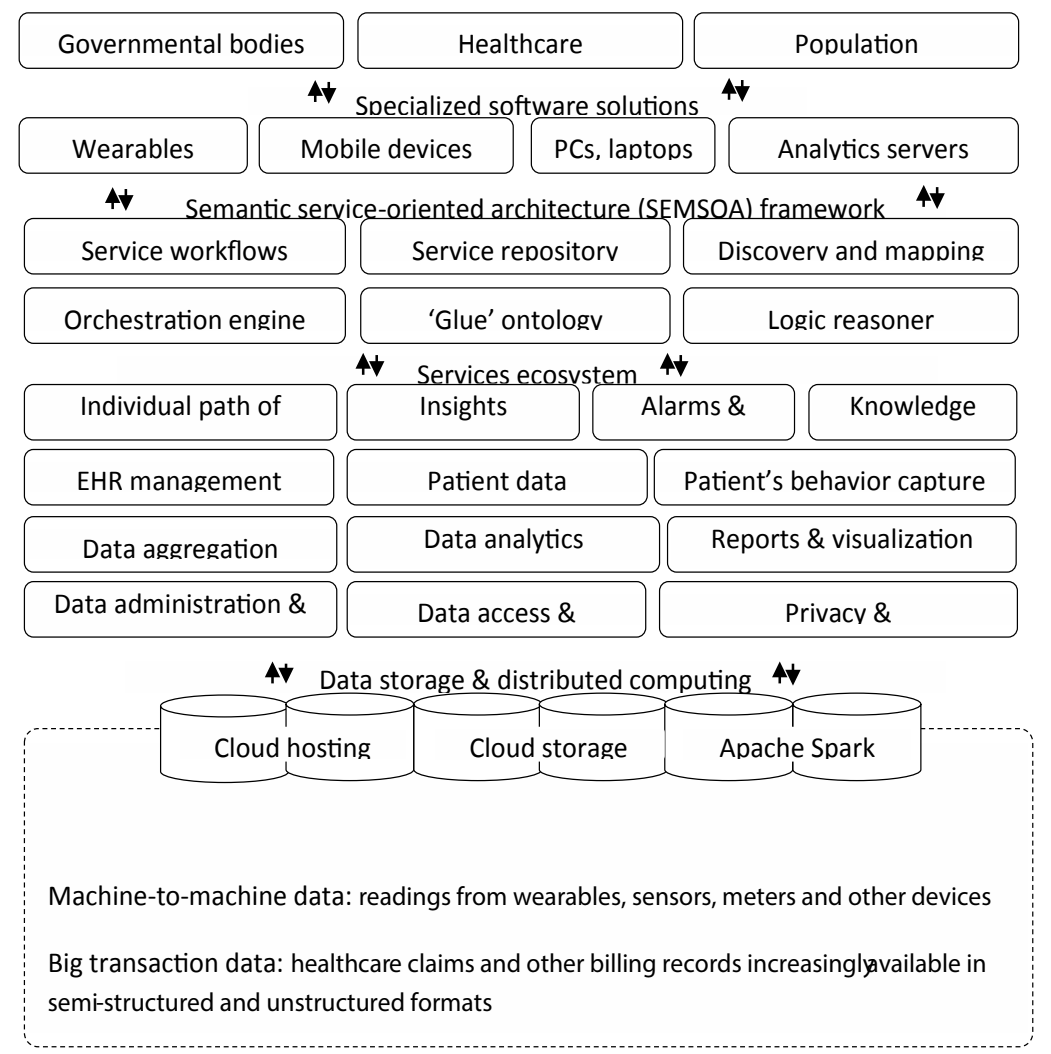

Figure 1: Key elements of the proposed architecture.

\section{Methodology}

The planned activities in particular include the following:

- Investigating a variety of healthcare-related data sources (EHR data, unstructured clinical notes, claims, medical imaging data, mobile devices output, other sectors data, social platforms and membership portals, etc.), comparing their feathers and providing means for converting all data to digital forms (say, by scanning paper documents), and developing ontologies for different data source classes.

- Studying existing and developing portable personal devices, used for biometrical data collection, comparing their features and parameters, and developing ontologies for different devices outcomes.

- Using behavioral and environmental data which are the newest and possibly fastest-growing in health care thanks to improving patients' monitoring by wearable or implantable sensors for capturing context of patients at any given time: their location (indoor / outdoor), activity (sleeping, walking, performing a particular task), emotional state (i.e. stress, depression, etc.), social state (i.e. degree of interaction, communication style, etc.) and environmental surroundings (weather conditions, air pollution, noises, etc.).

- Developing the repository of services which are related to processes of data collection and storing from all data sources (and developing the ontology of these services).

- Investigating a variety of healthcare-related data sources (EHR data, unstructured clinical notes, claims, medical imaging data, mobile devices output, other sectors data, mobile applications, social platforms and membership portals, etc.), comparing their feathers and providing means for converting all data to digital forms (say, by scanning paper documents), and developing ontologies for different data source classes.

- Studying portable personal devices, used for biometrical data collection, comparing their features and parameters, and developing ontologies for different devices outcomes.

- Using behavioral and environmental data which are the newest and possibly fastest-growing in health care thanks to improving patients' monitoring by wearable or implantable sensors for capturing context of patients at any given time: their location (indoor / outdoor), activity (sleeping, walking, performing a particular task), emotional state (i.e. stress, depression, etc.), social state (i.e. degree of interaction, communication style, etc.) and environmental surroundings (weather conditions, air pollution, noises, etc.).

- Developing the repository of services which are related to processes of data collection and storing from all data sources (and developing the ontology of these services).

- Developing the repository of services which are related to analytics processing of data across the healthcare ecosystem for extracting valuable intelligence from data (and developing the ontology of these services). They include also insights and inform actions for patients, care providers, health payers and insurers, and life science companies.

- Providing the mechanism of customer-driven medical applied 
software development by compositing and orchestrating dynamically discovered services from developed repositories to form the individual patient pathway (patient-specific workflows) of monitoring and treatment, taking into account different existing rules, regulations and standards.

- Providing opportunities for patients to communicate with others who have the same disease or condition, track and share their own experiences, see what treatments have helped other patients like them, gain insights and identify any patterns.

The main goals are the automation of the discovery of the devices and their data and the automation of mapping between different interfaces and data formats. This will make possible to create, improve, maintain and share medical data processing workflows expressed in the abstract terms of some 'glue' high-level ontology rather than in low-level details of interaction with concrete SOAP and REST web services. It is a far more flexible and scalable way which should boost the implementation of that smart ecosystem mentioned above.

Compared to FHIR tools, mentioned above, the proposed framework will be more about ontology development (that will help to integrate data formats, software tools, and data gathering devices) and mining the medical Big Data to contribute to the quality of the individual patient care and population health management. In this case people are more than patients. They are individual health consumers, and they are becoming empowered and engaged as healthcare becomes more personalized and convenient. With this model, patients become their own health managers. It seems like a perfect approach to reach the goal of every treatment - to involve all people's power to fight against the disorder and to predict its development and complications.

We are looking for partners who are interested to create jointly the Service-oriented "USE CASE" for supporting Big Data in eHealth with new levels of data exchange and interoperability by developing repositories of services, which are listed in the Table 1. The vision of micro-services architecture of the Service-oriented "USE CASE" is shown on Figure 2.

Technically the project will provide dynamic infrastructure of Complex healthcare "USE CASE" by developing the repository of semantically described interoperable services from which any individual patient pathway (patient-specific workflows) can be composed. Ontologies are used not only to integrate data formats, software tools as a service collection, gathering portable personal devices data and behavioural and environmental data, but to analytic processing of data across the healthcare ecosystem. Solutions had to be expanded across the health continuum, from healthy living, prevention, diagnosis, treatment, recovery and home care, to truly impact patients' health at the individual and population levels and to the most clinically and costeffective treatments to apply.

From this point of view any mobile application (for asthma, diabetes or other diseases), which will be developed, is only demonstration of way how to use developed ecosystem facilities (Figure 3).

\section{Conclusions}

The project is initiated for providing healthcare organizations with tailored Cloud ecosystem to support their Big Data analysis needs. Different data resources are integrated (EHR data, unstructured clinical notes, claims, medical imaging data, mobile devices output, other sectors data, social platforms and membership portals, etc.), comparing their feathers and providing means for converting all data to digital forms. Such huge amount of data is processing to develop personalized medicine and integrated solutions that support healthcare

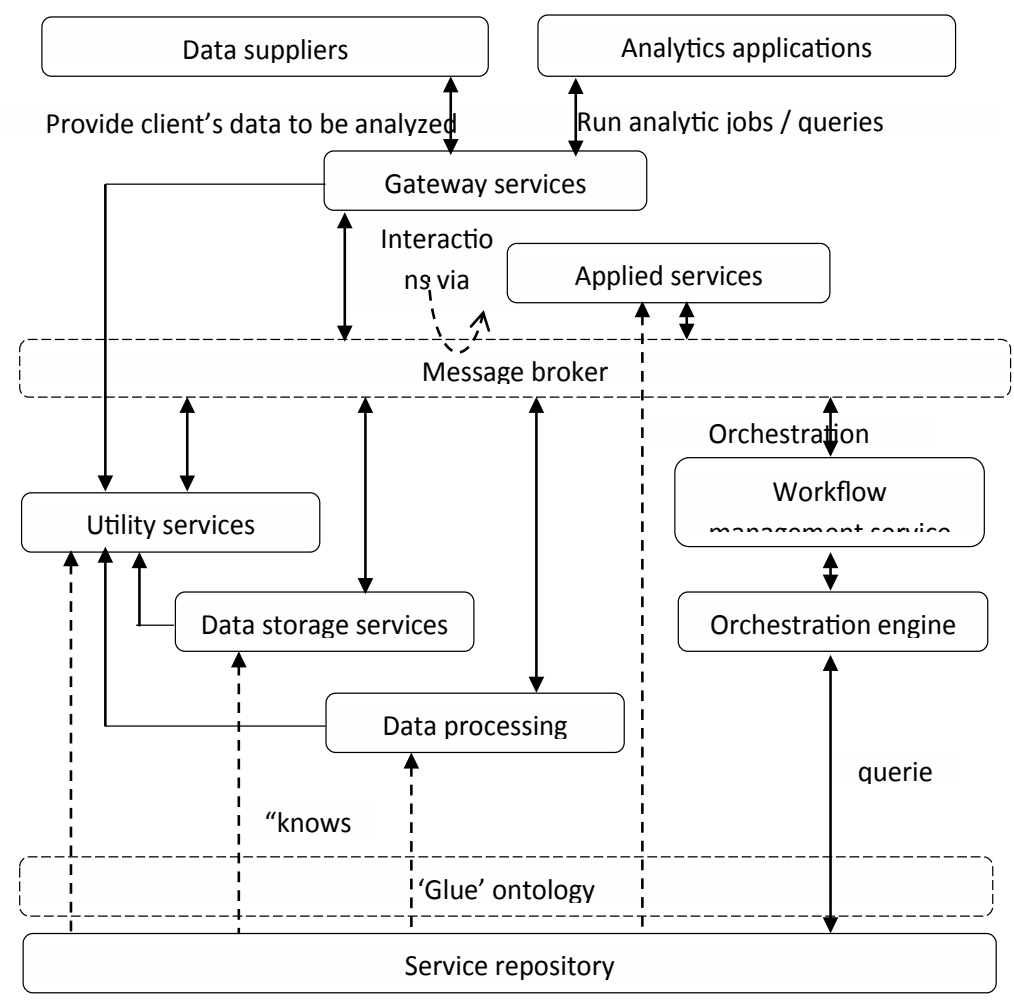

Figure 2: The Complex healthcare "USE CASE" micro-services architecture. 
Citation: Petrenko Al (2016) Services and a Distributed Ecosystem for Supporting Mobile Medical Application Development. J Telecommun Syst Manage 5: 140. doi: 10.4172/2167-0919.1000140

Page 6 of 7

\section{Use case contribution}

\section{Data and data resources}

Classification of portable devices indicators which turns usual smartphone into a portable medical diagnostic device or even in a laboratory setting (blood glucose meters, blood pressure monitors, peak flowmeter, heart rate meter, ECG monitor, bodily fluid colorimetric tester, liquid handling systems, activity measuring, ultrasound systems, otoscope, eye care tools, GPS inhalers or insulin pumps, environmental screening tools, etc.) and development their ontologies for interoperable usage together with a wireless transmitter that communicates with the sensor and a mobile medical app.

Forming services for picking up outputs of portable devices used for diabetes and asthma patients (an implanted sensor for constant remote glucose levels measuring acoustic detection technology for coughing and wheezing control and traditional spirometry with FEV1 or FEV1/FVC ratio; Allergic and non-allergic triggers estimation).

Evaluation and digitizing data of additional resources: Medical History (EPHR, Disruption of usual activities, Sleep disturbance, Adherence to medical treatment plan, Interval exacerbation of symptoms, Symptoms suggesting comorbid conditions or alternative diagnosis, Side effects of medications); Physical Examination (height, eyes, ears, nose, throat, lungs, heart, skin, X-ray, etc.); Context data (say, Asthma Triggers/Allergens), etc. and development their ontologies for interoperable usage.

\section{Data aggregating services}

Ontology-based services for gathering the various portable wearable devices output data.

Ontology-based services for managing the EHR data, clinical notes, claims, medical imaging data.

Ontology-based services for capturing the information of patients' behavior and environment parameters.

Copying (replication) data for improving their localization, increasing availability and reducing the risk of loss.

Using metadata (data about data) with descriptive information about the origin of the data, ways of generating (measured or calculated).

Content data management with Semantic support.

Data translation between standard and task-specific data exchange formats.

Access Control for managing authorization policies, and provides authorization decisions for requests to PGIDS data and services.

Aggregating and storing data of patients' personalized monitoring.

Cloud distributed data storage with multi-channel-multi-service access

Powerful associative data search and discovery (search by value, not by the location), and automatic parallel access.

Cloud Repository as a multi cloud application hosted platform which contains services and applications descriptions and supports consumers and developers of applications.

Application Mashup creator with a service composition editor and an execution engine for definition and execution of service workflows by special 'glue' high-level ontology. It is a distributed and fully decentralized and scalable and reliable tool for composite services

Context-aware adaptation of workflow processes

The individual patient pathway (patient-specific workflows) forming in the semantically-enhanced event-driven service-oriented architecture (SEMSOA).

Open Access to obtained results and an obligation to ensure that all publications produced will be linked to the Open portal.

Opening services code and algorithms made to make them to be available for and to (re)use by others.

Data Warehouse facilities for supporting management of open data at large scale and transform it into knowledge

Data processing services (Analytics)

Using EPHR for automatic circulation and accessibility of medical data about the patient, filling its fields and read data from EPHR

Remote monitoring of the patient's status in any place and at any time. Estimating patients risk of becoming ill or developing a serious condition.

Preparing the Treatment Plan (roadmap) and control its fulfilling, taking into account individual feathers of patients and their personal allergy records

Support for the electronic prescription (ePrescription)

Providing Extreme Warning and Emergency Information, when a doctor (and / or ambulance staff) will be immediately informed if vital patient's parameters get close to a dangerous point.

Insights synthesis and informing patients, health professionals, health payers and insurers, and life science companies' actions.

Integrating individual patients' data across a community into a broader, meaningful view of health and healthcare in a particular region to support healthcare migrating from episodic and fragmented illness response to a patient-centric model of public healthcare.

Data mining procedures for detecting hidden patterns of aggregated data in the form of significant features, correlations, and trends.

Complex event detection in distributed big data streams.

Examples of Application Development by Service Composition

A mobile application for patients with diabetes which uses wearable sensors data, texting information, location, movement information, and public environment data A mobile application for asthmatics which uses GPS-enabled monitoring inhaler, peakflowmetry data and information about asthma triggers presenting in patient's location Evidence-supported disease epidemiology (say, asthma), based on measuring of the disease feathers in time and space using GIS (geographic information system) and environment data as well as understanding the disease transmission, reservoir, immunity and treatment.

Table 1: Main services of the Complex eHealthcare "USE CASE". 


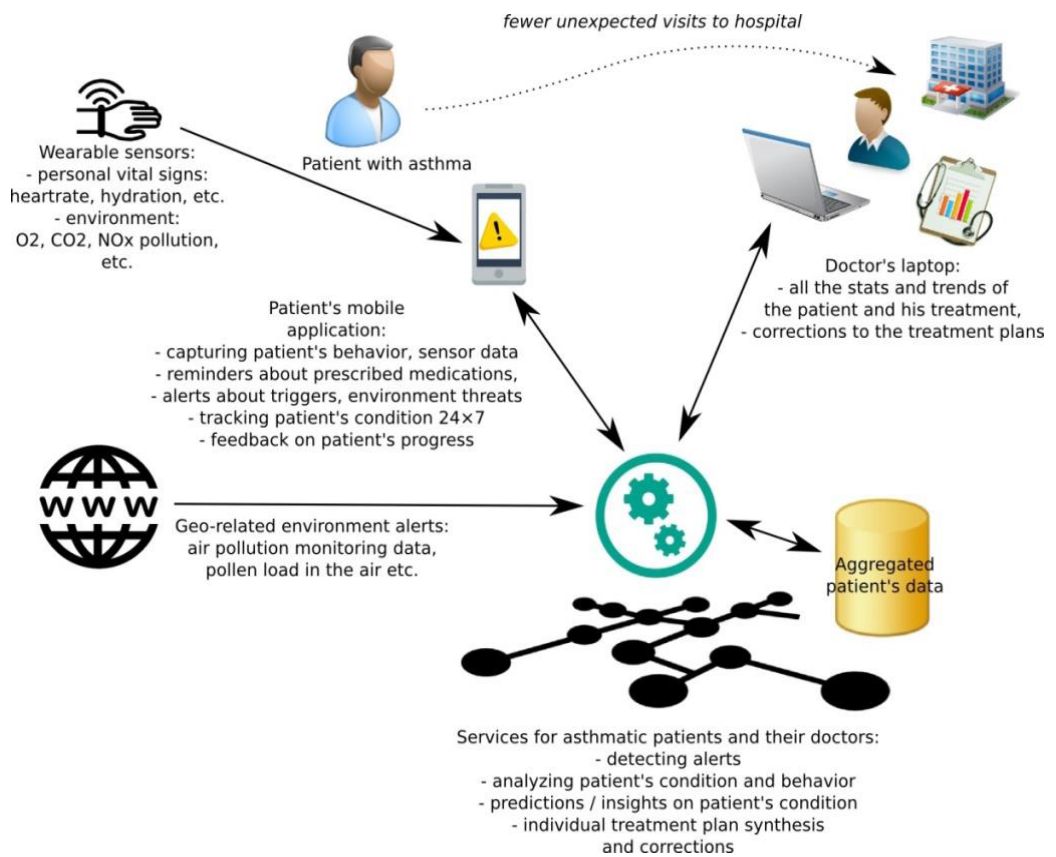

Figure 3: Demonstration of a mobile application scenario realisation for patients with asthma.

system management on public health policies and to provide actionable insights for patients at the point of care. Technically the project provides dynamic infrastructure by developing the repository of semantically described interoperable services from which any individual patient pathway (patient-specific workflows) can be composed [25]. Ontologies are used not only to integrate data formats, software tools as a service collection, gathering portable personal devices data and behavioural and environmental data, but to analytic processing of data across the healthcare ecosystem. The last ones include also insights and inform actions for patients, care providers, health payers and insurers, and life science companies. Events in the ecosystem with semanticallyenhanced event-driven service-oriented architecture (SEMSOA) can be initiated by patients, doctors, and devices outcomes. Solutions can be expanded across the health continuum, from healthy living, prevention, diagnosis, treatment, recovery and home care, to truly impact patients' health at the individual and population levels and to the most clinically and cost-effective treatments to apply.

\section{References}

1. https://ec.europa.eu/digital-single-market/en/public-consultation-green-papermobile-health

2. https://ec.europa.eu/digital-single-market/en/news/ehealth-action-plan-20122020-innovative-healthcare-21st-century

3. https://www.mulesoft.com/resources/api/connected-healthcare

4. http://ec.europa.eu/health/ehealth/docs/ev_20121107_wd02_en.pdf

5. http://portal.euro.who.int/en/ehealth-task-force-report-redesigning-health-ineurope-for-2020/

6. https://www.hl7.org/fhir/overview.html

7. http://www.citeulike.org/user/ruddyconsult/article/5961153

8. http://www.esens.eu/

9. https://ec.europa.eu/digital-single-market/en/news/inventory-cross-borderegovernment-services-analysis-existing-and-future-needs-and-demand-cross

10. https://ec.europa.eu/digital-single-market/en/content/action-91-memberstates-agree-common-list-key-cross-border-public-services
11. https://ec.europa.eu/digital-single-market/en/news/ehealth-interoperabilityframework-study-0

12. https://www.ihe.net/About_IHE/

13. http://www.healthcareitnews.com/directory/continua-health-alliance

14. http://www.epsos.eu/home/about-epsos.html

15. http://www.bsigroup.com/en-GB/about-bsi/uk-national-standards-body/ European-standardization-regulation/

16. http://www.eu-patient.eu/globalassets/projects/sustains/sustains_userrequirement-recommendations.pdf

17. http://ewma.org/what-we-do/ewma-project-portfolio/list-of-completed-ewmaprojects/united4health

18. http://www.semantichealthnet.eu/index.cfm/news/

19. https://ec.europa.eu/digital-single-market/en/connecting-europe-facility

20. https://www.healthcatalyst.com/healthcare-analytics-adoption-model/

21. https://www.google.com/int//en/health/about/

22. https://en.wikipedia.org/wiki/Microsoft_HealthVault

23. https://www-01.ibm.com/software/bd/industry/healthcare/

24. http://www.ibmbigdatahub.com/healthcare

25. https://rd-alliance.org/digital-infrastructures-research-28-30-september-2016kr\%C3\%A1kow 